\title{
On the BCH formula of Rezek and Kosloff
}

\section{Jan Naudts*, Winny O'Kelly de Galway}

Departement Fysica, Universiteit Antwerpen, Universiteitsplein 1, 2610 Antwerpen, Belgium

\section{A R T I C L E I N F O}

\section{Article history:}

Received 16 February 2011

Received in revised form 28 April 2011

Available online 5 May 2011

\section{Keywords:}

Baker-Campbell-Hausdorff formula

Quantum heat engine

Lie algebras

Density matrix

\begin{abstract}
A B S T R A C T
The BCH formula of Rezek and Kosloff (2006) [10] is a convenient tool to handle a family of density matrices, which occurs in the study of quantum heat engines. We prove the formula using a known argument from Lie theory.
\end{abstract}

(c) 2011 Elsevier B.V. All rights reserved.

\section{Introduction}

The Hamiltonian

$$
H=\frac{1}{2} \hbar \omega\left(a^{\dagger} a+a a^{\dagger}\right)
$$

of the quantum harmonic oscillator belongs to the Lie algebra $\mathfrak{s u}(1,1)$ with generators

$$
\begin{aligned}
& S_{1}=\frac{1}{4}\left(\left(a^{\dagger}\right)^{2}+a^{2}\right), \\
& S_{2}=\frac{i}{4}\left(\left(a^{\dagger}\right)^{2}-a^{2}\right), \\
& S_{3}=\frac{1}{4}\left(a^{\dagger} a+a a^{\dagger}\right) .
\end{aligned}
$$

As a consequence, it is possible to write down simplified Baker-Campbell-Hausdorff (BCH) relations [1,2]. These are used to study the quantum harmonic oscillator in a time-dependent external field [3-9]. The topic of the present paper is a new $\mathrm{BCH}$ relation, introduced recently by Rezek and Kosloff [10]. They consider the family of density matrices

$$
\rho=\frac{1}{Z(\beta, \gamma)} e^{\gamma a^{2}} e^{-\beta H} e^{\bar{\gamma}\left(a^{\dagger}\right)^{2}}
$$

with real $\beta$ and complex $\gamma$, and with

$$
Z(\beta, \gamma)=\operatorname{Tr} e^{\gamma a^{2}} e^{-\beta H} e^{\bar{\gamma}\left(a^{\dagger}\right)^{2}}
$$

\footnotetext{
* Corresponding author. Tel.: +32 32652459.

E-mail address: jan.naudts@ua.ac.be (J. Naudts).
} 
Since all operators appearing in (3) belong to the Lie algebra it is clear from the general Baker-Campbell-Hausdorff relation that it must be possible to write

$$
e^{\gamma a^{2}} e^{-\beta H} e^{\bar{\gamma}\left(a^{\dagger}\right)^{2}}=e^{\chi a^{2}-\xi H+\bar{\chi}\left(a^{\dagger}\right)^{2}} .
$$

The explicit expression of the coefficients $\chi$ and $\xi$ as a function of $\beta$ and $\gamma$ is found in the Appendix of Ref. [10]. The functions were derived [11] using the algebraic manipulation software Mathematica. Here, the same relations are derived by a simple analytic argument.

Note that the special case of (5) with $\xi=0$ appeared in the physics literature before (see Example I of Section II of Ref. [12]; see also Refs. [13,14]). In the present paper, relation (5) is derived using the argument of Ref. [12]. This relation is of interest in its own. But it is also very useful in the study of quasi-stationary processes $[10,15]$.

We derive the $\mathrm{BCH}$ formula in the next section. In the final section follows a short discussion.

\section{The identity}

The l.h.s. of (5) can be written in terms of the generators of the Lie algebra $\mathfrak{s u}(1,1)$ as

$$
e^{2 \gamma\left(S_{1}+i S_{2}\right)} e^{-2 \beta \hbar \omega S_{3}} e^{2 \bar{\gamma}\left(S_{1}-i S_{2}\right)} .
$$

These generators satisfy the commutation relations

$$
\begin{aligned}
& {\left[S_{1}, S_{2}\right]=i S_{3},} \\
& {\left[S_{2}, S_{3}\right]=-i S_{1},} \\
& {\left[S_{3}, S_{1}\right]=-i S_{2} .}
\end{aligned}
$$

Introduce $\mathfrak{s u}(2)$ generators $T_{1}=-i S_{1}, T_{2}=i S_{2}$, and $T_{3}=S_{3}$. Then (6) becomes

$$
X \equiv e^{2 \gamma\left(i T_{1}+T_{2}\right)} e^{-2 \beta h \omega T_{3}} e^{2 \bar{\gamma}\left(i T_{1}-T_{2}\right)} .
$$

The relation (5) does not depend on the choice of the representation of the $\mathfrak{s u}(2)$ algebra. Therefore, we may change it. A favourable choice is that of the Pauli spin matrices $\sigma_{\alpha}=2 T_{\alpha}$. Using that $\left(\sigma_{1} \pm i \sigma_{2}\right)^{2}=0$ and $\sigma_{\alpha}^{2}=\mathbb{I}$, the calculation becomes very easy. One obtains

$$
\begin{aligned}
X & =e^{i \gamma\left(\sigma_{1}-i \sigma_{2}\right)} e^{-\beta \hbar \omega \sigma_{3}} e^{i \bar{\gamma}\left(\sigma_{1}+i \sigma_{2}\right)} \\
& =\left(\mathbb{I}+i \gamma\left(\sigma_{1}-i \sigma_{2}\right)\right)\left(\cosh (\hbar \omega)-\sigma_{3} \sinh (\hbar \omega)\right)\left(\mathbb{I}+i \bar{\gamma}\left(\sigma_{1}+i \sigma_{2}\right)\right) \\
& =e^{-\beta \hbar \omega \sigma_{3}}-2 \kappa|\gamma|^{2}+i \kappa(\gamma+\bar{\gamma}) \sigma_{1}+\kappa(\gamma-\bar{\gamma}) \sigma_{2}+2 \kappa|\gamma|^{2} \sigma_{3},
\end{aligned}
$$

with $\kappa=e^{-\beta \hbar \omega}$ as before. On the other hand is

$$
\begin{aligned}
\exp \left(\chi a^{2}-\xi H+\bar{\chi}\left(a^{\dagger}\right)^{2}\right) & =\exp \left(2 \chi\left(S_{1}+i S_{2}\right)-2 \xi \hbar \omega S_{3}+2 \bar{\chi}\left(S_{1}-i S_{2}\right)\right) \\
& =\exp \left(2 \chi\left(i T_{1}+T_{2}\right)-2 \xi \hbar \omega T_{3}+2 \bar{\chi}\left(i T_{1}-T_{2}\right)\right) .
\end{aligned}
$$

In the Pauli spin representation this becomes $e^{Y}$ with

$$
Y=i(\chi+\bar{\chi}) \sigma_{1}+(\chi-\bar{\chi}) \sigma_{2}-\xi \hbar \omega \sigma_{3} .
$$

Since the Pauli matrices anti-commute and their squares equal $\mathbb{I}$ it follows that

$$
Y^{2}=\lambda^{2} \mathbb{I} \quad \text { with } \lambda=\sqrt{\xi^{2}(\hbar \omega)^{2}-4|\chi|^{2}} .
$$

Hence one obtains

$$
e^{Y}=\cosh (\lambda)+\frac{1}{\lambda} \sinh (\lambda) Y .
$$

Comparison with (9) gives the 4 conditions

$$
\begin{aligned}
& \cosh (\lambda)=\alpha+\kappa, \\
& \frac{1}{\lambda} \sinh (\lambda)(\chi+\bar{\chi})=\kappa(\gamma+\bar{\gamma}), \\
& \frac{1}{\lambda} \sinh (\lambda)(\chi-\bar{\chi})=\kappa(\gamma-\bar{\gamma}), \\
& \frac{1}{\lambda} \sinh (\lambda) \xi \hbar \omega=\alpha,
\end{aligned}
$$


with

$$
\begin{aligned}
\alpha & =\sinh (\beta \hbar \omega)-2 \kappa|\gamma|^{2} \\
& =\frac{1}{2 \kappa}\left[1-\kappa^{2}-4 \kappa|\gamma|^{2}\right] .
\end{aligned}
$$

The solution of these equations is

$$
\begin{aligned}
& \xi=\frac{\alpha}{\hbar \omega} \frac{\lambda}{\sinh (\lambda)}, \\
& \chi=\kappa \frac{\lambda}{\sinh (\lambda)} \gamma
\end{aligned}
$$

with

$$
\sinh (\lambda)=\sqrt{\alpha^{2}-4 \kappa^{2}|\gamma|^{2}}
$$

These results coincide with those found in the Appendix of Ref. [10].

Note that the expressions for $\xi$ and $\chi$ can be inverted easily. Given $\xi$ and $\chi$, one obtains $\lambda$ from (12). Then $\alpha$ follows by inverting (19). This gives

$$
\alpha=\hbar \omega \xi \frac{\sinh (\lambda)}{\lambda} .
$$

Next $\beta$ is obtained from (14)

$$
\kappa=\cosh (\lambda)-\alpha \text {. }
$$

Finally, $\gamma$ follows from $(20)$

$$
\gamma=\frac{\sinh (\lambda)}{\kappa \lambda} \chi .
$$

\section{Discussion}

The $\mathrm{BCH}$ relation of Rezek and Kosloff is somewhat special because it is written in a form suited for application to density matrices. Similar results found in the literature [1-9] aim at the calculation of time evolution operators and refer to similarity transformations, that is, to expressions of the form $e^{A} B e^{-A}$. But the l.h.s. of (5) is not a similarity transformation. This is precisely the reason why this $\mathrm{BCH}$ relation is of interest! The change of the spectrum implies that the average energy $\langle H\rangle=\operatorname{Tr} \rho H$ will depend on the value of the parameter $\gamma$. This dependence is essential in the context of heat engines.

\section{References}

[1] J. Wei, E. Norman, Lie algebraic solution of linear differential equations, J. Math. Phys. 4 (1963) 575-582.

[2] J. Wei, E. Norman, On global representations of the solutions of linear differential equations as a product of exponentials, Proc. Amer. Math. Soc. 15 (1964) 327-334.

[3] H.R. Lewis Jr., W.B. Riesenfeld, An exact quantum theory of the time-dependent harmonic oscillator and of a charged particle in a time-dependent electromagnetic field, J. Math. Phys. 10 (1969) 1458-1473.

[4] C.M.A. Dantas, I.A. Pedrosa, B. Baseia, Harmonic oscillator with time-dependent mass and frequency and a perturbative potential, Phys. Rev. A 45 (1992) 1320-1324.

[5] Kyu Hwang Yeon, Hyon Ju Kim, Chung In Um, Thomas F. George, Lakshmi N. Pandey, Wave function in the invariant representation and squeezed-state function of the time-dependent harmonic oscillator, Phys. Rev. A 50 (1994) 1035-1039.

[6] D.-Y.Song, Unitary relation between a harmonic oscillator of time-dependent frequency and a simple harmonic oscillator with and without an inversesquare potential, Phys. Rev. A 62 (2000) 014103.

[7] S. Deffner, E. Lutz, Nonequilibrium work distribution of a quantum harmonic oscillator, Phys. Rev. E 77 (2008) 021128

[8] M. Kuna, J. Naudts, General solutions of quantum mechanical equations of motion with time-dependent Hamiltonians: a Lie algebraic approach, Rep. Math. Phys. 65 (2010) 77-107.

[9] J. Naudts, W. O'Kelly de Galway, Analytic solutions for a three-level system in a time-dependent field, Physica D 240 (2011) $542-545$.

[10] Y. Rezek, R. Kosloff, Irreversible performance of a quantum harmonic heat engine, New J. Phys. 8 (2006) 83.

[11] Y. Rezek, Private communication, 2010.

[12] R. Gilmore, Baker-Campbell-Hausdorff formulas, J. Math. Phys. 15 (1974) 2090-2092.

[13] R.A. Fisher, M.M. Nieto, V.D. Sandberg, Impossibility of naively generalizing squeezed coherent states, Phys. Rev. D 29 (1984) $1107-1110$.

[14] D.R. Truax, Baker-Campbell-Hausdorff relations and unitarity of SU(2) and SU(1,1) squeeze operators, Phys. Rev. D 31 (1985) $1988-1991$.

[15] P. Salamon, K.H. Hoffmann, Y. Rezek, R. Kosloff, Maximum work in minimum time from a conservative quantum system, Phys. Chem. Chem. Phys. 11 (2009) 1027-1032. 